\title{
Squamous cell carcinoma: variation in dermatoscopic vascular features between well and non-well differentiated tumors
}

\author{
John Pyne MBBS BOptom (Hons) MMed ${ }^{1}$, Devendra Sapkota BSc M.D. ${ }^{1}$, \\ Jian Cheng Wong BSc (Hons) MStat ${ }^{2}$
}

${ }^{1}$ School of Medicine, The University of Queensland, Brisbane, Australia.

${ }^{2}$ School of Mathematics and Statistics, The University of New South Wales, Sydney, Australia.

Key words: squamous cell carcinoma, well, moderate, poor, differentiation, vascular

Citation: Pyne J, Sapkota D, Wong JC. Squamous cell carcinoma: variation on dermatoscopy vascular features between well and non-well differentiated tumors. Dermatol Pract Conc. 2012;2(4):5. http://dx.doi.org/10.5826/dpc.0204a05.

Received: April 6, 2012; Accepted: July 21, 2012; Published: October 31, 2012

Copyright: $@ 2012$ Pyne et al. This is an open-access article distributed under the terms of the Creative Commons Attribution License, which permits unrestricted use, distribution, and reproduction in any medium, provided the original author and source are credited.

Funding: None.

Competing interests: The authors have no conflicts of interest to disclose.

Drs. John Pyne, Devendra Sapkota and Mr. Jian Cheng Wong had full access to all of the data in the study and take responsibility for the integrity of the data and the accuracy of the data analysis.

Corresponding author: Dr John Pyne, 131 Ellesmere Rd, Gymea Bay NSW 2227, Australia. Tel. 61+414 750 625; Fax. 61+2+9525 3193. Email: j.pyne@uq.edu.au.

\footnotetext{
ABSTRACT Objective: Compare the dermatoscopy vascular features of well differentiated with non-well differentiated squamous cell carcinoma (SCC).

Design: A prospective study of 294 consecutive cases of histopathologically confirmed invasive SCC compared the dermatoscopic vascular features of well to combined moderate and poorly differentiated tumors. These features were recorded live directly from the patients and included: the percentage of pink in the tumor, the presence of branching, serpentine, dot, hairpin, glomerular and linear vessels, and the number of these vessel types present within each tumor. Vessel types were also reviewed by tumor depth in $1 \mathrm{~mm}$ increments.

Setting: Two medical practices in Sydney, Australia.

Patients: Eighty-six female and 208 male patients (29-95 years old).

Main outcome measure: Dermatoscopic vascular feature variation between grades of tumor differentiation and tumor depth.

Results: Of 294 invasive SCCs, $255(87 \%)$ were well differentiated, 32 (11\%) were moderately differentiated and $7(2 \%)$ were poorly differentiated. The percentage of pink areas within tumors varied between differentiation grades. The combined group of moderate and poorly differentiated tumors displayed more branching $(28 \%, \mathrm{P}<0.001)$ and serpentine $(62 \%, \mathrm{P}<0.005)$ blood vessels compared to well differentiated tumors ( $8 \%$ and $38 \%$, respectively). Moderate and poorly differentiated tumors displayed larger numbers of vessel types (3.3) compared to well differentiated tumors $(2.6, \mathrm{P}<0.01)$. Branching and serpentine vessels both increased in incidence with increasing tumor depth $(\mathrm{p}<0.05)$. Conclusion: Grades of tumor differentiation in SCC display varying dermatoscopic vascular features. The incidence of branching and serpentine vessels increases with increasing tumor depth and the shift towards poor differentiation.
} 


\section{Introduction}

Compared to well differentiated squamous cell carcinoma (SCC), moderate and poorly differentiated SCC behave in a more aggressive manner and portend a worse prognosis [1]. Tumor differentiation has recently been incorporated into the new American Joint Committee on Cancer Staging System for Cutaneous SCC [2]. Non-well differentiated SCCs have been reported as deeper tumors [3] with greater recurrence rates $[3,4]$. Poorly differentiated SCC on the ear or mucosal lip has an increased risk of metastasis [4,5]. Differentiation grade in SCC has been stated as an independent adjusted predictor for overall survival [6].

Tumor differentiation in SCC presents along a spectrum from well to moderate to poor differentiation, which is determined using histopathologic criteria originally described by Broders [7]. Broders' original grading system correlates biological behaviour with differentiation in four grades. Grade 1 is characterized by abundant keratinization, little nuclear anaplasia and less than $25 \%$ undifferentiated cells. These features extend on spectra through to Grade 4 where there is little or no keratinization, extensive nuclear anaplasia and greater than $75 \%$ undifferentiated cells. Concordance in the histopathological assessment of SCC may lack consistency, even between experienced observers [8,9]. Routine histopathology reporting, as used in this study, categorizes SCC into three grades: well, moderate and poorly differentiated.

Dermatoscopic assessment of invasive SCC is a relatively neglected area of published investigation. Cutaneous horn formation and prominent surface scale are clinical features of keratinization that commonly develop on keratinocytic tumors. Dermatoscopically identified white circles (which represent targetoid infilling of the follicular infundibulum by keratinocytic hyperplasia), can be observed as part of in situ and invasive SCC [10]. Vessels associated with invasive SCC are often surrounded by a white halo, which is characteristic of keratinocytic tumors [11]. Although pigmented blood breakdown products are frequently seen with invasive cutaneous SCC, the presence of dermatoscopically identifiable melanin pigment is rare $[12,13]$. We speculated that grade of differentiation in SCC may have characteristic dermatoscopic appearances determined more by vascular and keratinization features rather than pigmented structures. Vascular features were the focus of this study.

\section{Methods}

Data was collected from July 2009 to December 2010 from two medical practices in Sydney, Australia. The study was approved by the Ethics Committee from The University of Queensland, Brisbane, Australia. Throughout the study time window, all cases for excision where invasive SCC was the obvious diagnosis, or in the differential, were assessed for inclusion. Vascular features were recorded live directly from a patient by observer 1 , who then left the room. Observer 2 then immediately entered the same room to record data live directly from the same patient with the same dermatoscope.

\section{Exclusion criteria}

Exclusion criteria were applied with the intention of preventing cases entering the study with dermatoscopic features confounded by a range of external factors not due to the tumor alone. These exclusion criteria were: residual or recurrent tumors at sites of previous surgical intervention (including partial biopsies); tumors with any part involving mucosal surfaces, tattoos or juxtaposed to scars; sites of previous radiotherapy, photodynamic therapy, cryotherapy, laser ablation, or topical pharmacological therapy (either patient or clinician initiated); and collision situations between invasive SCC and any non-SCC related entity evident from clinical, dermoscopic or histopathological examination and all cases of keratoacanthoma diagnosed by histopathology. Keratoacanthomas have been observed with a higher incidence of branching vessels $(25 \%, \mathrm{n}=100)$ compared to invasive SCC $(11 \%, n=410$, unpublished data).

\section{Vascular features assessed}

The tumor vascular features examined included: the percentage of pink area within a tumor, recorded as either no pink, less than $50 \%$ pink, or greater than $50 \%$ pink within the tumor boundary and the presence of tumor vessels with dot, hairpin (loop), glomerular (coil), branching (arborizing), serpentine (curvilinear) and linear (no branches or curves) morphology. The number of these different vessel morphologies within the tumor boundary was also recorded.

After the application of exclusion criteria, the defined vascular features of the remaining cases were recorded with a Heine Delta $20^{\circledR}$ nonpolarized dermatoscope (Heine, Optotechnic GmbH, Herrsching, Germany). Recording was performed prior to anesthetic injection, excision and submission for routine histopathological examination. To avoid vessel compression and enhance image resolution, all data was recorded after transparent ultrasound gel was applied between the glass plate of the dermatoscope and the skin surface.

All excised tissue was submitted for routine histopathological confirmation using hematoxylin and eosin staining. Histopathology reports were used to exclude all entities other than invasive SCC from the study. Tumor thickness was measured in all cases to the nearest $0.1 \mathrm{~mm}$ and tabulated with the grade of either well, moderate or poor differentiation. Due to the low number of cases, moderate and poorly differentiated tumors were assessed combined. 
TABLE 1. Squamous cell carcinoma: tumor cases by depth $(\mathrm{mm})$ and grade of differentiation

\begin{tabular}{|l|c|c|c|c|c|}
\hline Tumor depth $\mathbf{( m m})$ & $<\mathbf{1 . 0}$ & $\mathbf{1 . 0 - 1 . 9}$ & $\mathbf{2 . 0 - 2 . 9}$ & $\mathbf{3 . 0 - 3 . 9}$ & $\mathbf{4 . 0}$ or $>$ \\
\hline Well differentiated SCC & $\mathrm{n}=124$ & $\mathrm{n}=66$ & $\mathrm{n}=46$ & $\mathrm{n}=10$ & $\mathrm{n}=5$ \\
Total cases n = 55 & $49.4 \%$ & $26.3 \%$ & $18.3 \%$ & $3.98 \%$ & $1.99 \%$ \\
Percentage & & & & & \\
\hline Moderately differentiated SCC & $\mathrm{n}=5$ & $\mathrm{n}=12$ & $\mathrm{n}=9$ & $\mathrm{n}=2$ & $\mathrm{n}=4$ \\
$\begin{array}{l}\text { Total cases } \mathrm{n}=32 \\
\text { Percentage }\end{array}$ & $16 \%$ & $38 \%$ & $28 \%$ & $6.1 \%$ & $13 \%$ \\
\hline $\begin{array}{l}\text { Poorly } \\
\text { differentiated SCC }\end{array}$ & $\mathrm{Nil}$ & $\mathrm{n}=2$ & $\mathrm{n}=1$ & $\mathrm{n}=2$ & $\mathrm{n}=2$ \\
Total cases n = & $0 \%$ & $2 \%$ & $1 \%$ & $2 \%$ & $2 \%$ \\
\hline
\end{tabular}

TABLE 2. Squamous cell carcinoma: tumor depth—range and mean by grade of differentiation

\begin{tabular}{|l|c|c|}
\hline Tumor Differentiation Grade & Range of Tumor Depth & Mean Tumor Depth \\
\hline $\begin{array}{l}\text { Well differentiated } \\
\mathrm{n}=255\end{array}$ & $0.1-7.5 \mathrm{~mm}$ & $1.3 \mathrm{~mm}$ \\
\hline $\begin{array}{l}\text { Moderate differentiated } \\
\mathrm{n}=32\end{array}$ & $0.7-6.0 \mathrm{~mm}$ & $2.3 \mathrm{~mm}$ \\
\hline $\begin{array}{l}\text { Poorly differentiated } \\
\mathrm{n}=7\end{array}$ & $1.5-6.3 \mathrm{~mm}$ & $3.6 \mathrm{~mm}$ \\
\hline
\end{tabular}

\section{Statistical assessment}

Interobserver agreement between two of the authors (JP and DS) was assessed. Kappa values from 0.41 to 0.60 indicate moderate agreement between observers, 0.61 to 0.80 are regarded as substantial agreement, and from 0.81 to 1.00 almost perfect. Confidence intervals were set at $95 \%$.

Logistic regression was carried out to test for significance of trends. Logistic regression was used to model binary response variables (i.e., vessel morphology observed or not observed) in terms of explanatory variables (tumor depth).

\section{Results}

A total of 294 cases entered the study based on histopathological confirmation of invasive SCC. These 294 cases were comprised of well differentiated $86.7 \%$ (255/294), moderately differentiated $10.9 \%$ (32/294), or poorly differentiated $2.4 \%$ (7/294) tumors. The age of patients ranged from 29 to 95 years, the median age was 72 years, the mean age 70.8 years, and $70.7 \%(208 / 294)$ were males.

Well differentiated tumors tend to present in higher proportions with less depth (Table 1). Compared to well differ- entiated tumors, moderate and poorly differentiated tumors tend to have a higher proportional incidence as tumor depth increases (Table 1). Maximum recorded tumor depth for each grade of differentiation was well $(7.5 \mathrm{~mm})$, moderate $(6.0$ $\mathrm{mm})$ and poor differentiated $(6.3 \mathrm{~mm})$; the range of tumor depth and mean depth by grade of tumor differentiation are displayed in Table 2 .

\section{Pink areas}

Examination of the data on the percentage of pink areas does not reveal any highly distinctive association between the grade of differentiation and the proportion of pink within the tumor (Figure 1). When reviewing the poorly differentiated tumor data alone, 5 out of these 7 cases had greater than $50 \%$ pink within the tumor boundary.

\section{Vessel morphology}

Moderate and poorly differentiated SCC displayed branching in $28.2 \%(11 / 39)$ CI $16.5-43.8$ and serpentine vessels in $61.5 \%(24 / 39)$ CI 45.9-75.1 compared to well differentiated SCC with $7.5 \%$ (19/255) CI 4.8-11.3 and 37.6\% (96/255) CI 31.9-43.7 respectively (Figure 2 ). In poorly differentiated tumors, 4 out of the 7 cases had branching vessels. Hair- 


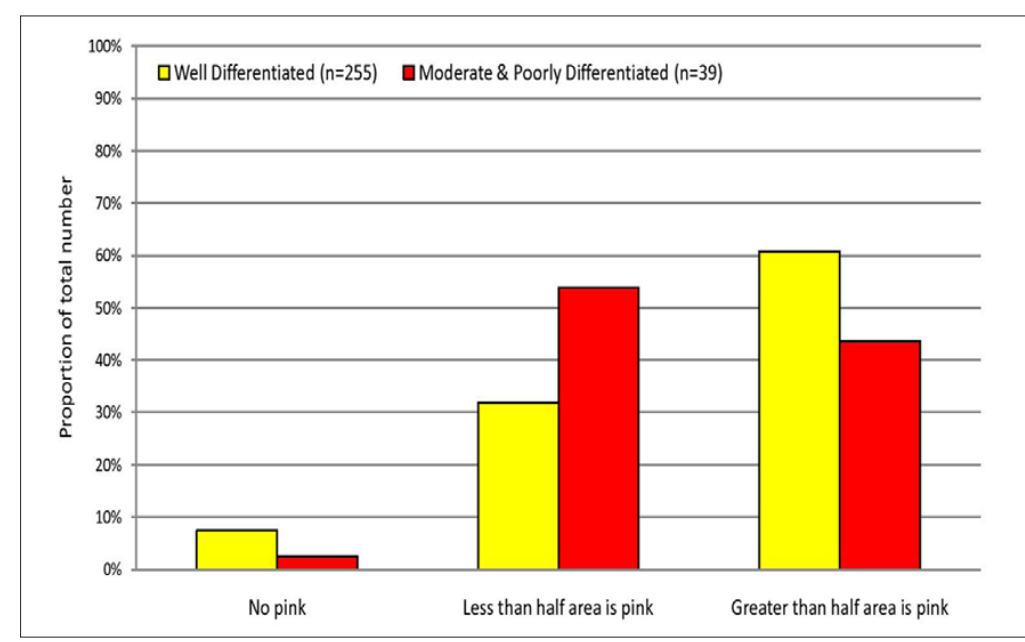

Figure 1. The percentage of pink in the tumor mass. [Copyright: (C2012 Pyne et al.]

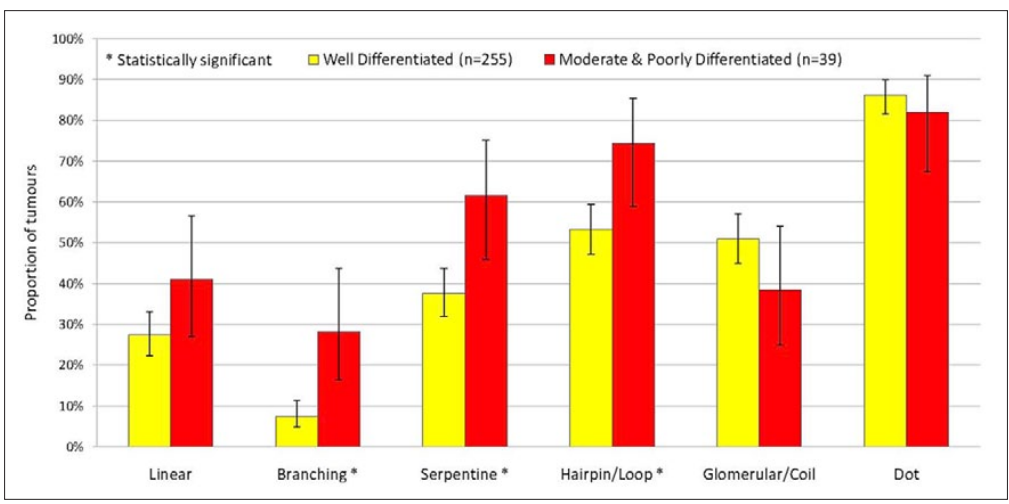

Figure 2. Comparison of different vessel morphologies with differentiation. [Copyright: (C2012 Pyne et al.]

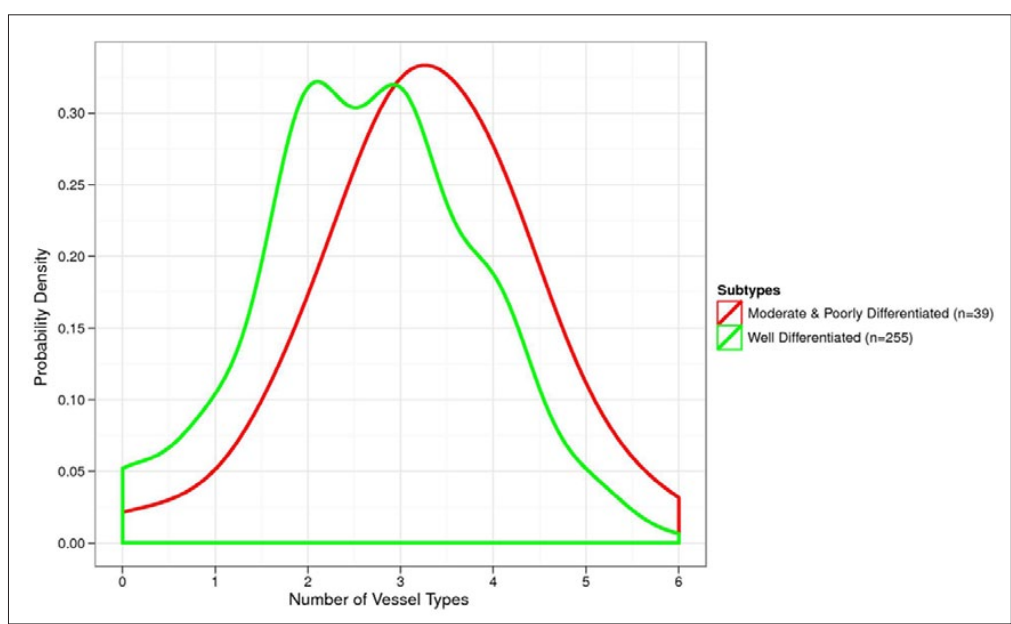

Figure 3. Vessel polymorphism by grade of tumor differentiation. A right shift for the probability density curves of moderate and poorly differentiated SCC compared to well differentiated SCC indicate that moderate and poorly differentiated tumors are more likely to display numerous vessel morphologies. The area under curve when number of vessel morphologies were larger or equal than 3 represents the total probability for 3 or more vessel morphologies being observed within the tumors. The moderate and poorly differentiated SCC had a larger area under curve (i.e., higher probability) compared to well differentiated SCC. The point at maximal probability represents the number of vessel morphologies being observed most frequently within the tumors. This number is also higher (a right shift) for moderate and poorly differentiated SCC compared to well differentiated SCC. [Copyright: (C2012 Pyne et al.] pin vessels were also significantly more frequent in moderate and poorly differentiated tumors. However, this has limited practical diagnostic value as the majority of well differentiated tumors also display hairpin vessels.

\section{Vessel polymorphism: number of vessel morphologies}

The number of different vessel morphologies present within the tumor mass as a function of tumor grade is displayed in Figure 3. For the study vessel morphologies selected, there is a significant increase in the average number of these vessel types in moderate and poorly differentiated tumors: 3.3 compared to 2.6 in well differentiated tumors, $\mathrm{P}<0.01$. Examples of vessel morphology and polymorphism among SCC with well, moderate and poor differentiation are shown (Figures 4-6). Of note, while well differentiated tumors commonly show a white halo (Figure 4), poorly differentiated tumors typically displayed vessels without a white halo (Figure 6).

\section{Vessel morphology variation with tumor differentiation and depth}

Branching, serpentine and linear vessel morphology records were each separately reviewed comparing well and non-well differentiated tumors by depth, as displayed in Figures 7,8 and 9 respectively. While both branching and serpentine vessels show an increase in incidence with depth, this trend is more pronounced in non-well differentiated tumors. For non-well differentiated SCC, branching and serpentine vessel incidence significantly increases with increasing tumor depth, $\mathrm{P}<0.05$. Well differentiated SCC also shows a trend of increasing branching and serpentine vessel incidence, however the significance of the trend is statistically weak. The trends in linear vessel are not statistically significant for both well and non-well differentiated SCC. 


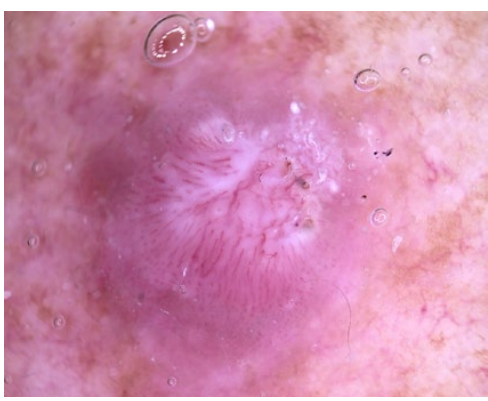

Figure 4. Dermatoscopy of a well differentiated SCC on the shoulder, orderly peripheral dot vessels merging into more central hairpin or loop vessels. The central tumor area has an elevated surface with white structureless areas. Vessels displaying a while halo are easily identified. [Copyright: (C2012 Pyne et al.]

\section{Interobserver agreement on classification by vessel morphology}

Kappa values for branching vessels $(0.81)$ and pink areas (0.80) were almost perfect. Serpentine (0.78) and hairpin (0.74) vessels were in substantial agreement (Table 3 ).

\section{Discussion}

Increased tumor depth was associated with a higher proportion of non-well differentiated SCC. This result is consistent with earlier work [3). Thicker non-well differentiated tumors often appeared endophytic and usually displayed a relatively low profile surface, and this was quite characteristic of poorly differentiated tumors. In practice, poorly differentiated SCC a deeper tumor depth may not be apparent from both clinical and dermatoscopy observation.

\section{Pink areas}

The study records of the crude percentage of pink in the tumor mass did not demonstrate convincingly obvious differences between the different differentiation grades. Pink areas in well differentiated tumors were often observed distributed relatively even around the periphery of the lesion. In non-well differentiated SCC, the associated pink

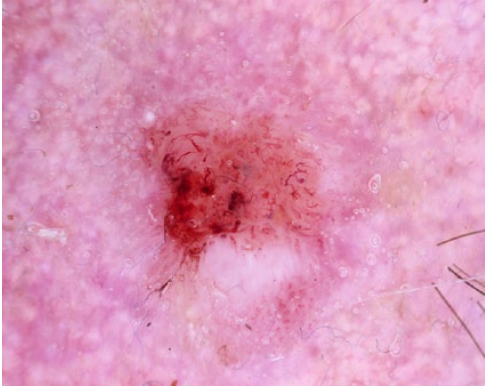

Figure 5. Dermatoscopy of a moderately differentiated SCC on the temple; flat pink central area with peripheral pink and white areas displaying asymmetric spatial distribution, bizarre vessel forms, numerous branching and serpentine vessels. [Copyright: (C2012 Pyne et al.]

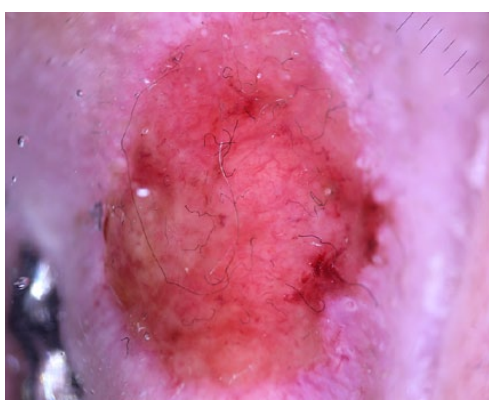

Figure 6. Dermatoscopy of a poorly differentiated SCC on the ear; the tumor surface follows the cartilage contours; pink areas cover the whole tumor surface; branching vessels without a white halo are common. [Copyright: (C2012 Pyne et al.]

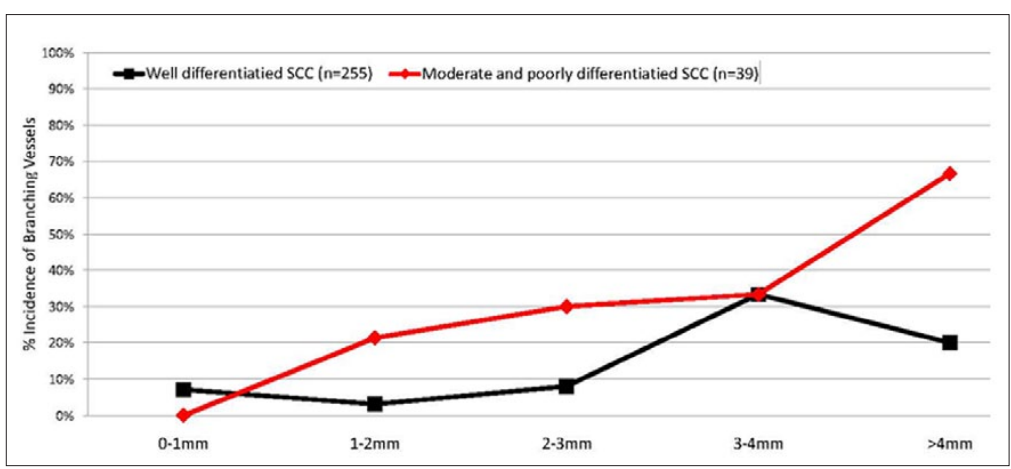

Figure 7. Branching vessels by tumor depth: well differentiated SCC verses non-well differentiated SCC . [Copyright: @2012 Pyne et al.]

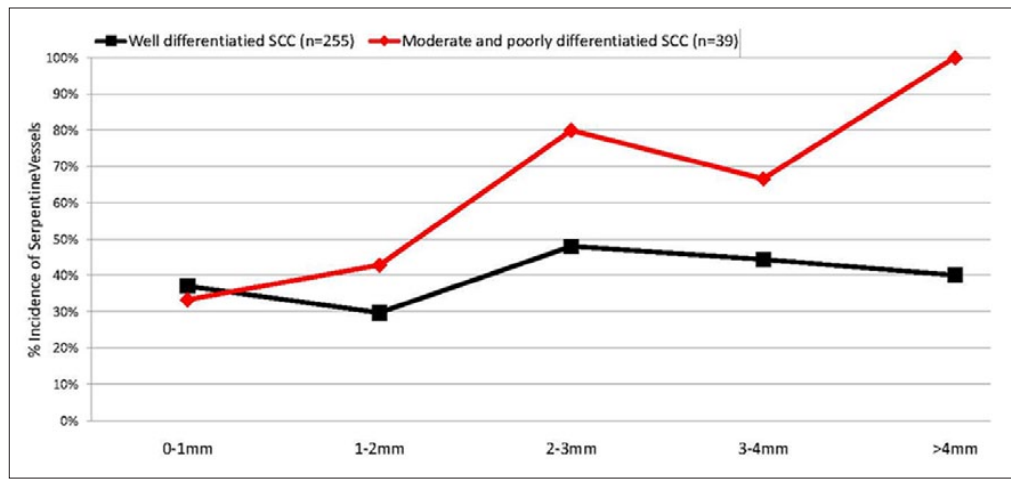

Figure 8. Serpentine vessels by tumor depth: well differentiated SCC verses non-well differentiated SCC. [Copyright: (02012 Pyne et al.]

areas were frequently central and in irregular spatial arrangements.

\section{Vessel types and polymorphism}

Well differentiated tumors had a relatively reduced incidence of branching and serpentine vessel forms. They were also characterized by dot, glomerular and hairpin vessels and fewer numbers of vessel types. Vessels commonly showed a white halo. Moderate and poorly differentiated tumors displayed a larger number of vessel types and frequently had irregular, bizarre vessel forms in irregular arrangements. Bizarre vessel forms can contribute to vessel 
TABLE 3. Squamous cell carcinoma: vascular feature Kappa values

\begin{tabular}{|l|c|c|c|}
\hline Vascular feature & $\begin{array}{c}\text { Cohen's Kappa } \\
\text { value }\end{array}$ & $\begin{array}{c}\text { Confidence Interval } \\
\text { Lower bound }\end{array}$ & $\begin{array}{c}\text { Confidence Interval } \\
\text { Upper Bound }\end{array}$ \\
\hline Branching vessels & 0.83 & 0.67 & 0.99 \\
\hline Serpentine vessels & 0.78 & 0.49 & 1.00 \\
\hline Hairpin vessels & 0.74 & 0.57 & 0.91 \\
\hline Glomerular vessels & 0.87 & 0.75 & 0.99 \\
\hline Dot vessels & 0.66 & 0.45 & 0.87 \\
\hline Linear vessels & 1.00 & 1.00 & 1.00 \\
\hline Pink areas & 0.80 & 0.58 & 1.00 \\
\hline
\end{tabular}

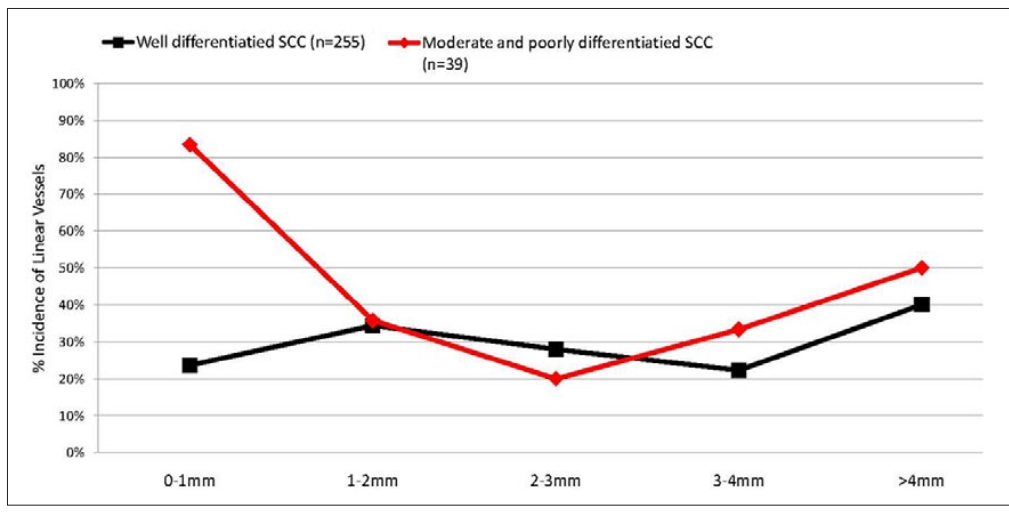

Figure 9. Linear vessels by tumor depth: well differentiated SCC verses non-well differentiated SCC. [Copyright: @2012 Pyne et al.]

polymorphism; however, this aspect of vessel form variation was not incorporated into the data records. In addition, poorly differentiated tumors typically displayed vessels without a white halo.

Vessel morphology variation with tumor differentiation and depth

Branching and serpentine vessel incidences increased with increasing tumor depth for both well and non-well differentiated tumors; this trend was more pronounced for moderate and poorly differentiated tumors compared to well differentiated tumors. Linear vessels did not display this trend.
Major limitations of this study include a small number of poorly differentiated SCCs ( $\mathrm{n}=7)$, moderately differentiated SCC ( $\mathrm{n}=32)$, and the reviewing and analysis of moderately and poorly differentiated tumors combined together. The use of polarized dermatoscopy may not produce the same findings as the study non-polarized dermatoscopy. The presence or absence of ulceration was not recorded in the study data. Tumor ulceration may alter the vessel features.

Future investigation of non-well differentiated SCC exploring the spatial distribution of vessel types and the spa- tial variation in pink within the tumor boundary correlated to the immediate histopathology may reveal useful information relevant to practice. The incidence of perivascular white halos in well differentiated compared to poorly differentiated tumors also deserves further investigation.

\section{Conclusions}

Dermatoscopy features correlating with the grade of differentiation in SCC will be expected to vary depending on the proportion and spatial location of areas of specific differentiation within a tumor mass. However, various vascular features may serve as indicators to the grade of differentiation in SCC.

This study found that the shift from well towards poor differentiation in SCC is represented by an increase in the incidence of branching and serpentine vessels that increased with tumor depth and an increase in vascular polymorphism.

\section{Acknowledgement}

The statistical analysis in this study was made by Mr. Wong and Dr. Pyne. Drs. Pyne, Sapkota and Mr. Wong contributed to the analysis and interpretation of data. 


\section{References}

1. Cassarino DS, Derienzo DP, Barr RJ. Cutaneous squamous cell carcinoma: a comprehensive clinicopathological classification. Part one. J Cutan Pathol. 2006;33(3):191-206.

2. Farasat S, Yu SS, Neel VA, et al. A new American Joint Committee on Cancer staging system for cutaneous squamous cell carcinoma: Creation and rationale for inclusion of tumor (T) characteristics. J Am Acad Dermatol. 2011;64(6):1051-9.

3. Jensen V, Prasad AR, Smith A, et al. Prognostic criteria for squamous cell cancer of the skin. J Surg Res. 2010:159(1): 509-16.

4. Eroglu A, Berberoglu U, Berreroglu S. Risk factors related to locoregional recurrence in squamous cell carcinoma of the skin. J Surg Oncol 1996;61(2):124-30.

5. Samarasinghe V, Madan V, Lear JT. Management of high-risk squamous cell carcinoma of the skin. Expert Rev Anticancer Ther. 2001;11(5): 763-9.

6. Kyrgidis A, Tzellos TG, Kechagias N, et al. Cutaneous squamous cell carcinoma (SCC) of the head and neck: risk factors of overall and recurrence-free survival. Eur J Cancer. 2010:46(9):1563-72.

7. Broders AC. Practical points on the microscopic grading of carcinoma. NY State J Med. 1932;32:667-71.
8. Davis DA, Donahue JP, Bost JE, Horn TD. The diagnostic concordance of actinic keratosis and squamous cell carcinoma. J Cutan Pathol. 2005;32(8):546-51.

9. Jagdeo J, Weinstock MA, Piepkorn M, Bingham SF; Department of Veteran Affairs Topical Tretinoin Chemoprevention Trial Group. Reliability of the histopathology diagnosis of keratinocyte carcinomas. J Am Acad Dermatol. 2007;57(2):279-84.

10. Zalaudek I, Giacomel J, Schmid K, et al. Dermatoscopy of actinic keratosis, intraepidermal carcinoma and invasive squamous cell carcinoma: A progression model. J Am Acad Dermatol. 2012;66(4):589-97.

11. Zalaudek I, Giacomel J, Leinweber B. Squamous cell carcinoma including actinic keratosis, Bowen's Disease, keratoacanthoma, and its pigmented variants. In: Soyer HP, Argenziano G, Hofmann-Wellenhof R, Johr R (eds.). Color Atlas of Melanocytic Lesions of the Skin. Berlin Heidelberg: Springer, 2007:295-302.

12. Sattler EK. Pigmented squamous cell carcinoma. Am J Dermatopathol. 2007;29(5): 486-9.

13. de Giorgi V, Alfaioli B, Papi F, et al. Dermoscopy in pigmented squamous cell carcinoma. J Cutan Med Surg. 2009; 13(6): 326-9. 